Julio- Diciembre 2017

"liseles de ni lijese

Revista de Investigación

Año 10

Académica sin Frontera

Núm. 26

ISSN: 2007-8870

http://revistainvestigacionacademicasinfrontera.com

Recibido el 17 de junio de 2017

Dictaminado mediante arbitraje favorablemente 10 de octubre de 2017

\title{
TRANSFERENCIA DE TECNOLOGIA UNIVERSIDAD-PYMES
}

\author{
IRMA CECILIA TRASVIÑA SOTO ${ }^{1}$ \\ DR. JUAN DE DIOS LEON LARA ${ }^{2}$ \\ DR. JAIME OLEA MIRANDA ${ }^{3}$ \\ DR. MIGUEL ANGEL CELIS ${ }^{4}$
}

\section{RESUMEN}

Para que México sea competitivo industrialmente, necesita establecer e impulsar actividades de investigación a través de programas multidisciplinarios, donde los representantes de las industrias e investigadores de la universidades, atiendan las diferentes problemáticas relacionadas con la producción, poniendo énfasis en la búsqueda de soluciones para lograr la modernización de la planta productiva, lo cual redundará en un mejoramiento de la calidad, en elevar la producción y bajar los costos. Este documento presenta los primeros avances del proyecto de investigación que tiene como objetivo medir la influencia de la transferencia de tecnología Universidad-Pymes en la competitividad empresarial que perciben los empresarios de las Pymes de Hermosillo, Sonora. Para lograr el objetivo propuesto se llevó a cabo una metodología mixta, la primera fase consistió en un análisis exploratorio, entrevistando a un grupo de seis expertos utilizando el método Delphi, con el fin de contextualizar el problema objeto de análisis y validación del instrumento medida; y en la segunda fase, se tomaron muestras de 71 gerentes de las Pymes. Los principales resultados en el avance de investigación, muestran que más del 90\% de los empresarios están conscientes de la importancia que tiene la vinculación Universidad-Pyme como fuente de innovación para sus negocios y que el logro de la competitividad empresarial se basa en definir de forma efectiva una estrategia o plan de I+D en sus negocios.

Palabras Clave: Aplicación de Conocimiento, Generación de Conocimiento, Transferencia de Tecnología.

\footnotetext{
${ }^{1}$ Departamento de Contabilidad, Universidad de Sonora, Blvd. Luis Encinas y Rosales s/n, Colonia Centro, Hermosillo, Sonora Tel.01(642)2592211 y 12, correo electrónico: ctrasvina@eca.uson.mx

2 Departamento de Ingeniería Industrial, Universidad de Sonora, Blvd. Luis Encinas y Rosales s/n, Colonia Centro, Hermosillo, Sonora, México Tel.01(662)259.22.11 jaime.olea@unison.mx

3 Departamento de Ingeniería Industrial, Universidad de Sonora, Blvd. Luis Encinas y Rosales s/n, Colonia Centro, Hermosillo, Sonora, México Tel.01(662)259.22.11 jleon@industrial.uson.mx

${ }^{4}$ Posgrado UPAEP, Puebla, Puebla, México macelis@sitecmexico.com.mx
} 


\title{
http://revistainvestigacionacademicasinfrontera.com
}

\begin{abstract}
In order for Mexico to be industrially competitive, it needs to establish and promote research activities through multidisciplinary programs, where representatives of the industries and researchers of the universities, attend to the different problems related to production, emphasizing the search for solutions to achieve the modernization of the production plant, which will lead to improved quality, increased production and lower costs. This document presents the first advances of the research project that aims to measure the influence of the University-SME technology transfer in the business competitiveness perceived by SME entrepreneurs in Hermosillo, Sonora. To achieve the proposed objective, a mixed methodology was carried out, the first phase consisted of an exploratory analysis, interviewing a group of six experts using the Delphi method, in order to contextualize the problem being analyzed and validation of the measured instrument; and in the second phase, 71 managers from the SMEs were sampled. The main results in the research progress show that more than $90 \%$ of entrepreneurs are aware of the importance of the link between University and SME as a source
\end{abstract}

Key words: Application of Knowledge, Knowledge Generation, Technology Transfer.

\section{Introducción}

Desde el comienzo del siglo actual ha aumentado el uso de diferentes tecnologías en los diversos ámbitos de la vida cotidiana, y especialmente en las empresas como una herramienta complementaria para aumentar la productividad y por consiguiente hacer de las organizaciones, empresas altamente competitivas. Según el Consejo Nacional de Ciencia y Tecnología (Conacyt, 2011), la Transferencia de Tecnología se define como la trasmisión formal y organizada de conocimientos apropiados para fabricar un producto, aplicar un procedimiento o la prestación de un servicio resultado de la actividad inventiva de una entidad a otra, teniendo como principal finalidad generar productos y servicios de alta calidad y de alto valor agregado; generando ventajas competitivas para la empresa dentro del mercado donde se desenvuelve y dándole la posibilidad de poder expandirse a otros nichos de mercado. 


\section{http://revistainvestigacionacademicasinfrontera.com}

Es indudable que el desarrollo científico y tecnológico es un factor potencial para el crecimiento económico y social de cualquier país. De manera que, el valor productivo que está inmerso se basa en el desarrollo de nuevos conocimientos e innovaciones, producto de procesos de investigación orientados a incrementar la capacidad tecnológica. En este sentido, las universidades tienen un rol protagónico, al ser el centro de mayor concentración de potencial de investigación, por lo tanto, se convierten en medios de apoyo a la sociedad para impulsar su crecimiento económico (Loaiza, Giugni, Monguet, 2009).

Para las Pymes es una realidad la transferencia de tecnología para crecer y desarrollar mejor sus productos y/o servicios; cabe mencionar que hoy en día ya existen patentes mexicanas generadas gracias a la Transferencia de Tecnología, utilizada como estrategia en muchas empresas (Cortes y Peñaloza, 2011). Del mismo modo, las formas de Transferencia de tecnología, están evolucionando pasando de la tradicional trasmisión de conocimientos planteados a las trasferencias acompañadas de estrategias comerciales, de inversión o de adquisición de negocios, llegando al nivel de la constitución de alianzas estratégicas. Sin embargo, la propia debilidad de la mayoría de las empresas de los países en desarrollo para participar en este tipo de alianzas, hace que persista en estos países la modalidad de análisis, desde el punto de vista del adquirente o receptor de una licencia.

Al respecto Aguilar y Martínez (2013), mencionan que la carencia de recursos tecnológicos en las Pymes es uno de los problemas más comunes, ya que no tienen el capital suficiente para invertir en maquinaria nueva y con tecnología lo cual no les permite mejorar sus procesos de producción, las ventas son insuficientes, debilidad competitiva, problemas en la gestión de impuestos y falta de financiamiento entre otros. Otro problema que actualmente enfrentan las pymes, es la globalización, que es el proceso a gran escala de la información, las tecnologías, la disminución de costos y transporte, consiguiendo que las empresas se establezcan 


\section{Revista de Investigación Académica sin Frontera ISSN: 2007-8870}

\section{http://revistainvestigacionacademicasinfrontera.com}

en otros países siendo aún más competitivas y que se eliminen trabas al comercio internacional (Soto, 2003).

La transferencia de tecnología Universidad-Pyme es la solución a la problemática actual de las Pymes, y su aplicación es el compromiso permanente para aumentar su competitividad mientras contribuyen activamente al desarrollo sostenible de la sociedad mediante acciones concretas y medibles dirigidas a solucionar los problemas prioritarios del país. Este documento presentar los primeros avances del proyecto de investigación que tiene por objetivo medir la influencia de la transferencia de tecnología Universidad-Pymes en la competitividad empresarial que perciben los empresarios de las Pymes de Hermosillo, Sonora.

\section{Marco Teórico-Conceptual}

2.1 Situación actual de las Pymes

Las Pymes son un estado evolutivo dentro del "ser empresario" y en América Latina más del 90\% de las empresas son Pymes, lo cual las presenta como el sector de mayor creación de empleo y notable participación en el Producto Interno Bruto (PIB), pero también con la mayor incidencia de fracaso (Sepúlveda, 2005).

La situación actual de las Pymes mexicanas -y de América Latina en general- no es la idónea: no tienen un alto grado de sobrevivencia ya que el 75\% desaparece apenas después de los 2 años de creadas (Red de Centros Regionales para la Competitividad Empresarial, México, 2009). Esto requiere, por supuesto, un análisis más profundo de las características y problemas de las pequeñas y medianas empresas mexicanas y su entorno, como las que plantea Gómez (2007):

- Tienen capital proporcionado por una o dos personas que establecen una sociedad.

- Los dueños dirigen la empresa. Son empresas familiares, la toma de decisiones depende de ellos.

- La administración es empírica

- Utilizan maquinaria y equipo, aunque se basen en el trabajo más que en el capital.

- Obtienen algunas ventajas fiscales. 


\section{http://revistainvestigacionacademicasinfrontera.com}

- Falta de liquidez y solvencia.

- Bajos niveles de productividad.

- Acceso restringido a fuentes de financiamiento.

Según Nacional Financiera (2015), el 43\% de los motivos o causas de fracaso son por problemas administrativos; el $16 \%$ por problemas fiscales; el $15 \%$ por la incapacidad de cumplir requisitos; el $11 \%$ por deficiencias en la comercialización; $8 \%$ por errores en insumos y producción; y el 7\% por la falta de financiamiento.

Hernández (2007), menciona que los obstáculos que tienen las Pymes se debe principalmente a fallas de los empresarios, que en su mayoría no cuentan con los conocimientos administrativos necesarios, ni con un soporte que le permita garantizar la obtención de créditos, y por consiguiente limitan la adquisición de tecnología, desconocen completamente el mercado que los margina, lo cual les complica el posesionarse del mismo, es más difícil el competir, conseguir nuevos clientes que cada día son más y más exigentes.

\subsection{Innovación tecnológica y su impacto en las Pymes}

Hoy en día la innovación juega un papel relevante en las estrategias empresariales para superar los retos del mercado globalizado. Ruiz (2008), señala que se ha vuelto crucial generar valor agregado para diferenciar productos, incluyendo el diseño innovador, el mercadeo eficaz, la distribución eficiente y las marcas comerciales acreditadas. De este modo, la industria que desea sobrevivir en el mercado al que pertenece, debe ser capaz de contribuir de manera productiva a cadenas de valor mundiales y generar nuevas cadenas de valor, de las cuales la parte clave no es necesariamente la producción, sino la innovación y los servicios de alto valor. Así Assink (2006), señala que el objeto de la innovación en la empresa se puede clasificar como cosas (productos y servicios) o como cambios en la manera en que creamos y entregamos productos y servicios (procesos). 


\section{http://revistainvestigacionacademicasinfrontera.com}

Con respecto a las Pymes no se puede ser excluyente, sino todo lo contrario, ya que observando más allá de su potencial en la creación de empleo y en la contribución a la producción de los países, las Pymes pueden tener un papel más importante en la innovación exitosa de productos y procesos, y en la creación de valor agregado asociado con ésta. Así las Pymes innovadoras son más sustentables y cuentan con ventajas competitivas que facilitan su competencia a nivel internacional. Adicionalmente, las Pymes innovadoras cuentan con mayores niveles de valor agregado y productividad, lo que aumenta su contribución a la competitividad de la economía en su conjunto (Canales, et al. 2008).

La innovación es esencial para la competitividad de las empresas, su importancia aumenta debido a factores tales como el incremento de la competencia global, la disminución de los ciclos de vida de productos y las demandas cambiantes de los consumidores (Storey, 2000). Las grandes empresas desempeñan un papel clave en la innovación, pero esto no quiere decir que no hay lugar para las Pymes, ya que, en los últimos años, el entorno de la innovación ha cambiado, favoreciendo y desarrollando el incentivo de creación de Pymes (Markatou, 2012).

Innovar en las Pymes significa mejorar en la eficiencia operativa a través de inversión en tecnologías de producción, incrementando así los ingresos por productos nuevos o mejorados. Esta se puede aplicar de dos formas en el mercado, la primera es la forma radical, que permite modificar sustancialmente los productos que realizan determinadas funciones. La segunda es la forma incremental que está constituida por las mejoras de las técnicas ya existentes en el proceso de fabricación de los productos. (Ruiz y Mandado, 1989). La innovación ha sido considerada como un factor importante para la creación y el mantenimiento de la competitividad de las naciones y las empresas, es por esto que para las Pymes es crucial una gestión empresarial basada en la aplicación de estrategias de innovación dentro de la planeación de la organización (Vargas y Guillen, 2005). 


\section{http://revistainvestigacionacademicasinfrontera.com}

\subsection{La transferencia de tecnología y su papel en la competitividad empresarial de las Pymes}

Es indudable que el desarrollo científico y tecnológico es un factor potencial para el crecimiento económico y social de cualquier país. De manera que, el valor productivo que está inmerso se basa en el desarrollo de nuevos conocimientos e innovaciones, producto de procesos de investigación orientados a incrementar la capacidad tecnológica. En este sentido, las universidades tienen un rol protagónico, al ser el centro de mayor concentración de potencial de investigación, por lo tanto, se convierten en medios de apoyo a la sociedad para impulsar su crecimiento económico (Loaiza, Giugni, Monguet, 2009).

Las empresas que tienen la necesidad de ser más eficientes en el uso de los recursos científicos y tecnológicos han generado la colaboración entre competidores, proveedores y otras instituciones públicas y privadas, incluyendo a las universidades y, busca completar el proceso de innovación para la producción de bienes y servicios de manera competitiva (Solleiro, 2008).

Por su parte, las universidades, como organismos de producción de conocimiento científico y en algunos casos tecnológicos, han intensificado sus actividades para activar relaciones con el sector empresarial. La estrecha relación ente el crecimiento de las industrias y el proceso de transferencia de tecnología incentivado por las universidades de cara a la industria, a partir de ese momento la innovación comercializada desde las universidades ha incrementado ostensiblemente (Pedraza y Velázquez, 2013). Dicho fenómeno no solo se produce en países industrializados, también en otros países donde las universidades tienen una estructura de derechos de propiedad intelectual similar, por ejemplo, Italia o el Reino Unido (Algieri et al., 2011).

Hoy en día, el crecimiento empresarial dentro del actual contexto mundial requiere gestión efectiva del conocimiento y de la innovación tecnológica que permita la creación de capacidades productivas, y sobre todo tecnológicas (Camacho, 2008). Solleiro y Castañón (2008), consideran la tecnología como una de las fuentes principales de la competitividad empresarial, ya que integra conocimiento, experiencia, equipo, instalaciones y software, y 


\section{http://revistainvestigacionacademicasinfrontera.com}

permite la generación de nuevos productos, procesos, servicios y sistemas, así como mejoras a lo que ya existen.

\section{Diseño de la investigación}

Dentro del trabajo de investigación se desarrollan una serie de actuaciones realizadas bajo dos fases de una investigación mixta. En una primera fase cualitativa se llevó a cabo un análisis exploratorio apoyada en un panel de expertos (conocido también como método Delphi), con el propósito de validar el instrumento de medida, y la segunda, la encuesta a expertos con la ayuda de un cuestionario semi-estructurado. El cuestionario tiene como objetivo, que basada en la opinión de los expertos se pueda medir la influencia de la transferencia tecnológica UniversidadPymes en la competitividad empresarial que perciben los empresarios de las Pymes de Hermosillo, Sonora.

\subsection{Elección y tipo de muestra}

Se tomará como base la información referente a las 385 Pymes registradas en el SIEM para el municipio de Hermosillo, Sonora. Hubo la necesidad de efectuar una depuración, ya que se discriminan las empresas que tengan las siguientes condiciones: a) duplicadas, b) maquiladoras, empresas y franquicias transnacionales y, c) Cadenas Comerciales Nacionales. Una vez efectuado este análisis, se obtuvo el tamaño real de la población de Pymes que fue de 272 empresas con las características del estudio, el cual se consideró como población para determinar la muestra, utilizando la fórmula para el muestreo aleatorio simple sin reemplazo, porque todos los elementos de la población tendrán la misma probabilidad de ser elegidos: 


\section{http://revistainvestigacionacademicasinfrontera.com}

En donde:

$$
n=\frac{\mathrm{N} * Z_{\alpha}^{2} * p *(1-p)}{\mathrm{e}^{2} *(N-1)+Z_{\alpha}^{2} * p *(1-p)}
$$

La población, $\mathrm{N}=272$

$\mathrm{Z} \alpha=0.05=1.96$, para un nivel de confianza del 95\%

Alfa $=\alpha=0.5$

$\mathrm{p}=0.5$

Error de la muestra $=\mathrm{e}=10 \%=0.1$

Nivel de confianza $=1-\alpha$

$$
\eta=71
$$

Por ende, ante el resultado obtenido con base en la aplicación de la formula anteriormente planteada se requieren recopilar 71 encuestas de la muestra de la población objeto de análisis, la cual está conformada por empresarios Pymes.

\subsection{Instrumento de recolección de datos}

Para poder realizar la comparación de las respuestas y por ende su categorización y generalización, se utilizará la entrevista abierta apoyada por un cuestionario semi-estructurado que contiene tres variables: 1) Generación de conocimiento (con 7 ítems), 2) Aplicación de conocimiento (con 9 ítems) y, 3) Competitividad empresarial (con 6 ítems), debido a que el cuestionario tiende a la estandarización, lo que permitirá comparar las respuestas y por ende su categorización y generalización. La información recopilada, organizada y ordenada, será capturada en el programa estadístico SPSS (Statistical Package for the Social Sciences), para su valoración e interpretación. 
Núm. 26

ISSN: 2007-8870

\section{http://revistainvestigacionacademicasinfrontera.com}

\section{Presentación, análisis e interpretación de resultados}

Se presentan a continuación los avances más relevantes basados en el objetivo central de esta investigación:

\subsection{Variable 1) Generación del conocimiento}

Imagen 1; Colaboración Universidad-Pyme

\section{La colaboración con la Universidad-Pyme, es una fuente de innovación y conocimiento.}

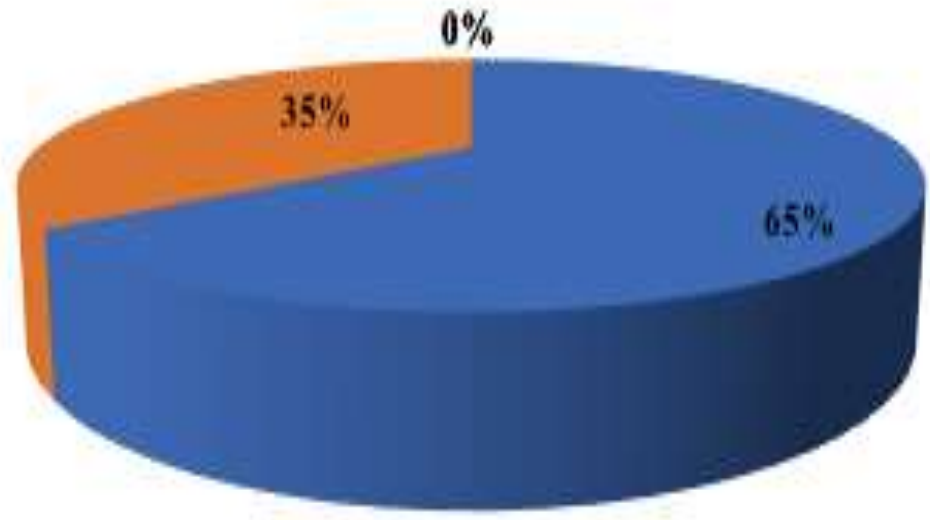

5. Siempre

4. Casi siempre

1I. Algunas veces

2. Casi nunca

1. Nunca

Fuente: Elaboración propia con base en los resultados estadísticos.

La imagen 1 muestra que el $65 \%$ de los empresarios de la muestra analizada opinan que estan consientes que la colaboración Universidad-Pyme es siempre una fuente de innovación y el $35 \%$ opina que casi siempre lo es. Estos resultados hacen ver que el empresario Pyme, posiblemente ya está consciente de que las condiciones del entorno empresarial de hoy, donde la alta complejidad y la fuerte competitividad se imponen, la gestión del conocimiento en la 
Núm. 26

ISSN: 2007-8870

\section{http://revistainvestigacionacademicasinfrontera.com}

empresa, en un elemento valioso para asegurar la sostenibilidad en el tiempo de los negocios y empresas del mundo.

Imagen 2; Conocimientos y capacidades del personal que genera conocimiento en la Universidad

Los conocimientos y capacidades de las personas que generan el conocimiento al interior de la Universidad-Pyme son imprescindibles para la generación de proyectos productivos para las Pymes.

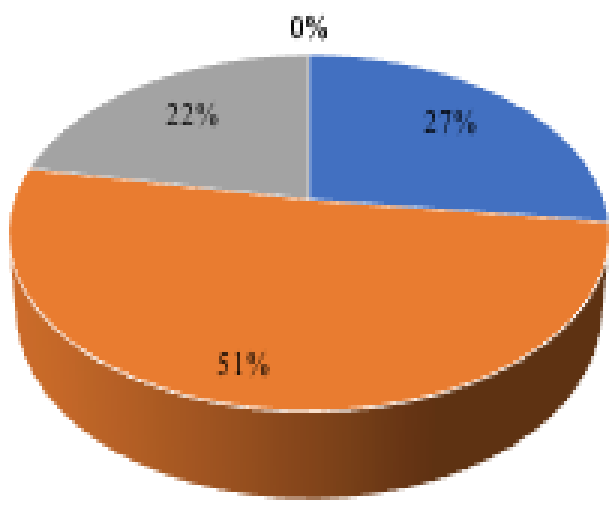

-5. Siempre

14. Casi siempre

3. Algunas veces

12. Casi nunca

-1. Nunca

Fuente: Elaboración propia con base en los resultados estadísticos

La imagen 2, muestra que el 51\% de los empresarios Pymes opinan que los conocimientos y capacidades de las personas que generan el conocimiento al interior de la universidad casi siempre son imprescindibles para la generación de proyectos productivos para las Pymes, el 27\% opina que siempre lo son y el $22 \%$ opina que algunas veces lo son. Los resultados muestran que el empresario esta consciente que es imprescindible que el personal que genera conocimiento en la universidad, lo anterior quizás porque sabe que si estas personas cuentan con la habilidad para obtener información y transformarla en un conocimiento útil que pueda ser rápidamente incorporado en la organización para después, ponerlo en práctica por esta, esto les permitirá tener la confianza de poder tener la mejor ventaja para hacer frente a la competencia y el reto de atender mejor a los clientes. 
Núm. 26

ISSN: 2007-8870

\section{http://revistainvestigacionacademicasinfrontera.com}

Imagen 3; Beneficios de la generación de proyectos productivos

\section{La generación de Proyectos Productivos Universidad-Pymes, beneficia a la empresa.}

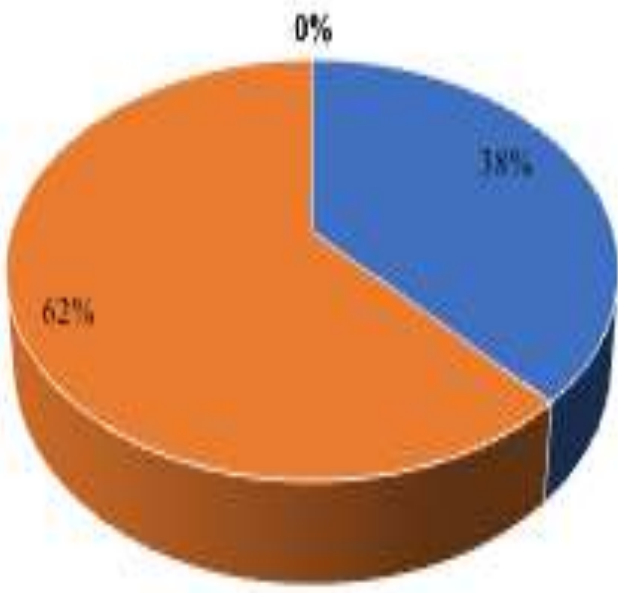

-5. Siempre

"4. Cusi siempre

3. Algunasveces

2. Casi nunca

-1. Nunca

Fuente: Elaboración propia con base en los resultados estadísticos

Los resultados de la imagen 3, muestra que el $62 \%$ de los empresarios opinan que la generación de proyectos productivos siempre trae beneficios a la empresa, el $28 \%$ opina que casi siempre les beneficia. Estos resultados hacen ver que los motivos que explican esta correlación se deben a que una correcta ges tión del conocimiento en la empresa facilita una gestión empresarial más eficiente. 
Núm. 26

ISSN: 2007-8870

\section{http://revistainvestigacionacademicasinfrontera.com}

Imagen 4; Importancia de generar conocimiento para las necesidades básicas de la población

La generación de conocimiento es imprescindible para alcanzar la meta de satisfacer las necesidades básicas de la población.

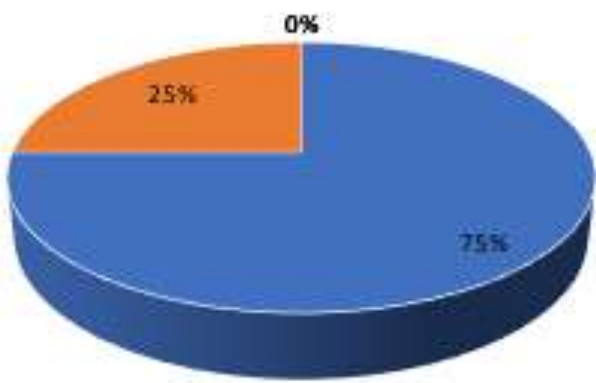

$$
\begin{aligned}
& \text {-5. Siempre } \\
& \text { " 4. Casi siempre } \\
& \text { "3. Algunas veces } \\
& \text { "2. Casi nunca } \\
& \text { "1. Nunca }
\end{aligned}
$$

Fuente: Elaboración propia con base en los resultados estadísticos

La imagen 4, muestra que el $75 \%$ de los empresarios de la muestra analizada opina que es imprescindible siempre la generación del conocimiento para alcanzar la meta de satisfacer las necesidades básicas de la población y el $25 \%$ opina que casi siempre es imprescindible. Los resultados hacen ver que el empresario está consciente que la ciencia y la tecnología hoy en día se han convertido en ramas de la actividad inseparables de la vida y el progreso de la sociedad.

Imagen 5; Satisfacción de la empresa con la utilidad que le genera la transferencia de conocimiento

\section{La empresa está satisfecha con la utilidad del conocimiento trasferido por parte de la Universidad.}

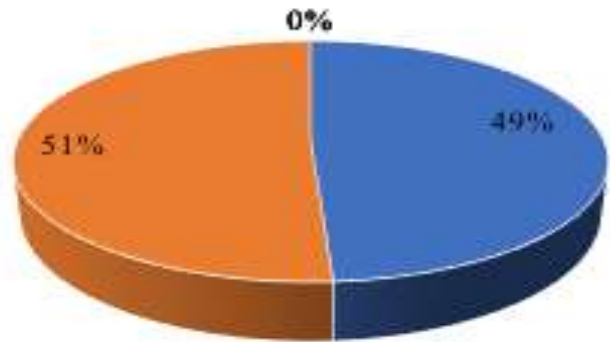

-5. Siempre

- 4. Casi siempre

"3. Algunas veces

"2. Casi nunca

-1. Nunca

Fuente: Elaboración propia con base en los resultados estadísticos 


\section{$\underline{\text { http://revistainvestigacionacademicasinfrontera.com }}$}

Dentro de los resultados de la imagen 5, los resultados muestran que el 51\% de los empresarios opinan que la empresa esta satisfecha casi siempre con el conocimiento transferido por parte de la universidad y el $49 \%$ siempre esta satisfecha. Lo anterior hace ver que la empresa esta satisfecha con la transferencia de conocimiento generado por la universidad a la empresa.

\subsection{Variable 2) Aplicación del conocimiento}

Imagen 6; Estrategia o plan de I+D por parte de la empresa

Tiene la empresa definida una estrategia o plan de $I+D$.
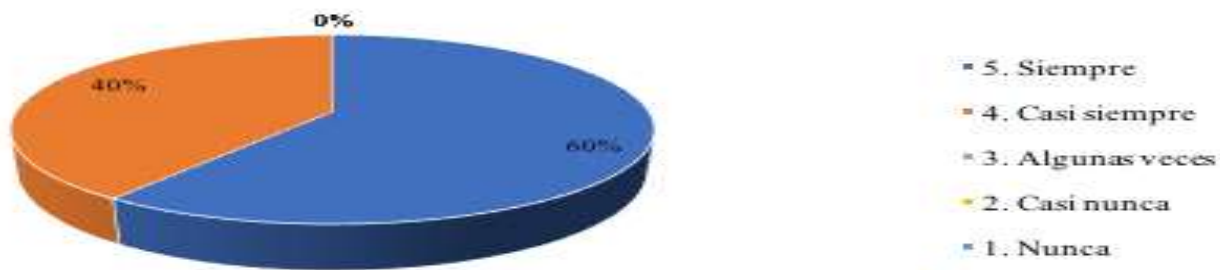

Fuente: Elaboración propia con base en los resultados estadísticos

La imagen 6 muestra que el 60\% de los empresarios de las Pymes de la muestra analizada tienen definida siempre una estrategia o plan de I+D en sus negocios, el $40 \%$ casi siempre la tienen. Estos resultados muestran que al ser empresas en crecimiento para ellos es imprescindible la definición de estrategias basadas en la I+D. 
Núm. 26

ISSN: 2007-8870

\section{http://revistainvestigacionacademicasinfrontera.com}

Imagen 7; La participación en proyecctos de I+D genera innovación y conocimiento en la empresa

\section{La participación en proyectos de I+D es una fuente de innovación y} conocimiento para la empresa.

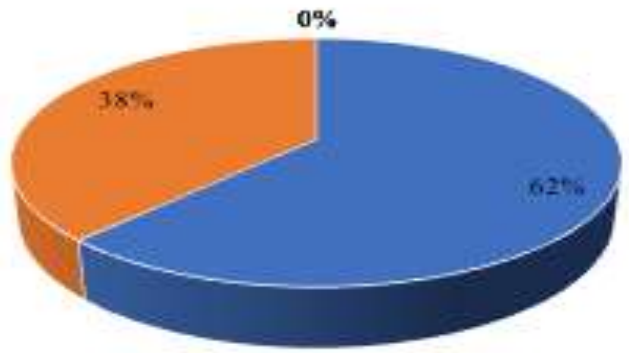

$$
\begin{aligned}
& \text {-5. Siempre } \\
& \text { "4. Casi siempre } \\
& \text { "3. Algunas veces } \\
& \text {-2. Casi nunca } \\
& \text { "1. Nunca }
\end{aligned}
$$

Fuente: Elaboración propia con base en los resultados estadísticos

Los resultados de la imagen 7, muestran que el 62\% de los empresarios opinan que la participación en proyectos de $\mathrm{I}+\mathrm{D}$ es una fuente de innovación y conocimiento para la empresa, el 38\% casi siempre. Estos resultados muetran que introducir la gestión del conocimiento en la empresa, tiene la virtud de transformar poco a poco sus estructuras rígidas en elementos más flexibles, capaces de dotar a la empresa de la fuerza necesaria para trasformar amenazas en oportunidades con base en I+D.

Imagen 8; La transferencia de tecnología frente a los competidores

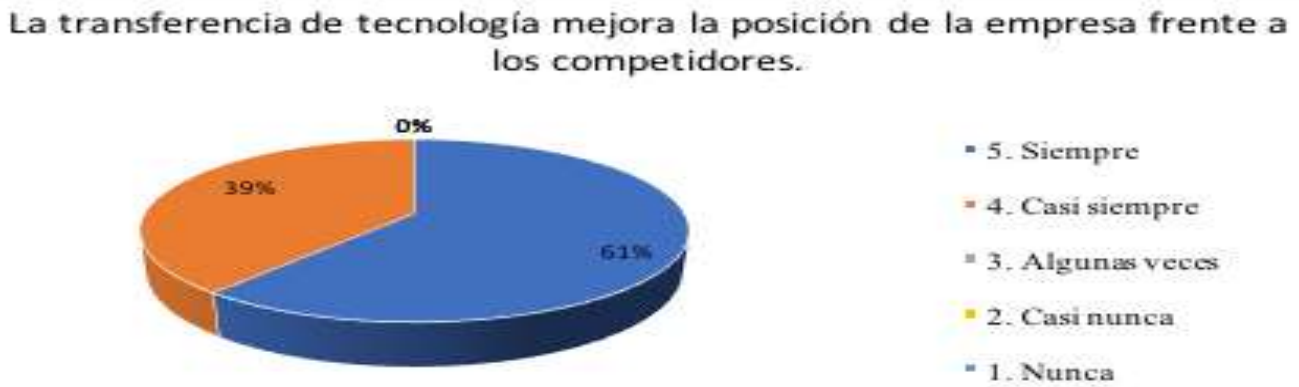

Fuente: Elaboración propia con base en los resultados estadísticos 


\section{$\underline{\text { http://revistainvestigacionacademicasinfrontera.com }}$}

En la imagen 8, los resultados describe que el $61 \%$ de los empresarios están conscientes de que la transferencia de tecnología es de gran ayuda porque mejora la posición de la empresa frente a los competidores y el 39\% opina que casi siempre la mejora. Los resultados muestran que la transferencia de tecnología Universidad- Pymes, permite a estas empresas a gestionar el cambio y poder de esta forma desarrollar organizaciones inteligentes que sean capaces de adaptarse a su entorno competitivo.

Imagen 9; Tranferencia de tecnología y la mejora en producto y/o servicio

\section{La transferencia de tecnología mejora la producción u oferta de productos y/o servicios}

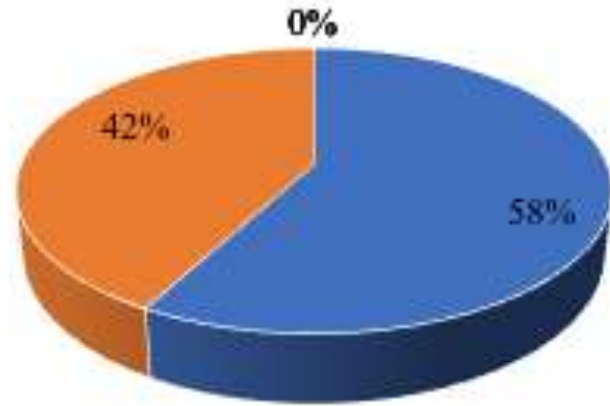

-5. Siempre

" 4. Casi siempre

11. Algunas veces

2. Casi nunca

=1. Nunca

Fuente: Elaboración propia con base en los resultados estadísticos

Los resultados de la imagen 9, muestran las opiniones de los empresarios y el $58 \%$ opina que la transferencia de tecnología mejora la producción y/o servico de sus negocios y el $42 \%$ opina que casi siempre. Lo anterior hace ver que la transferencia de tecnología puede ayudar a crear estrategias para mejorar los procesos internos de la empresa que nos permita lograr la eficiencia. 
Núm. 26

ISSN: 2007-8870

\section{http://revistainvestigacionacademicasinfrontera.com}

Imagen 10; Facilidad para la identificación de herramientas para la aplicación de proyectos productivos

\section{Hasta qué punto ha sido fácil para la empresa identificar que herramientas usar para el desarrollo de cada actividad, tarea o proceso}

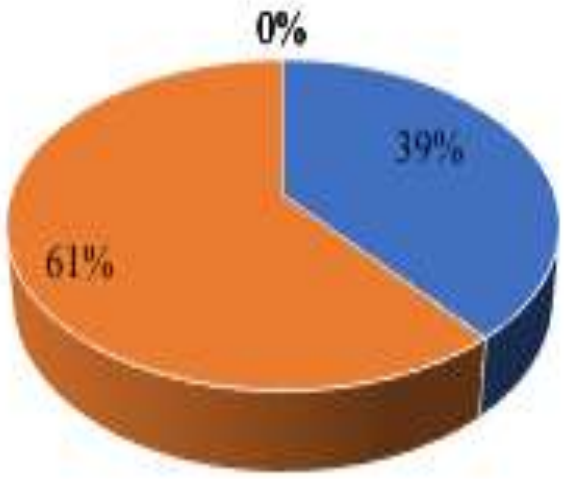

-5. Siempre

4. Casi siempre

1. Algunas veces

2. Casi nunca

-1. Nunca

Fuente: Elaboración propia con base en los resultados estadísticos

En la imagen 10, el 61\% de los empresarios opina que contar con una transferencia de tecnología Universidad- Pymes casi siempre le facilita a la empresa identificar que herramientas usar para el desarrollo de cada actividad, tarea o proceso y para el 39\% siempre les ha sido fácil. Lo anterior hace ver que aquellas empresas que buscan obtener ventaja competitiva estan conscientes que deben disponer de habilidad para obtener información, transformarla en conocimientos e incorporar este en la organización. 
Núm. 26

ISSN: 2007-8870

\section{http://revistainvestigacionacademicasinfrontera.com}

\subsection{Variable 3) Competitividad Empresarial}

Imagen 11; Existencia de un sistema de seguimiento para la competitividad empresarial

Construcción de un sistema de seguimiento que ayude a lograr de forma efectiva la competitividad de la empresa.

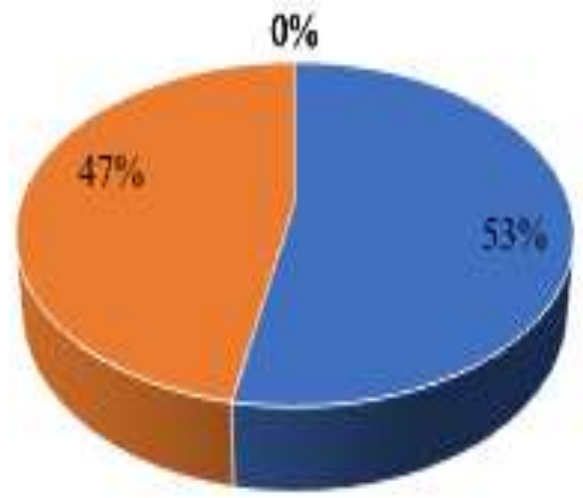

-5. Siempre

4. Casi siempre

-3. Algunas veces

-2. Casi nunca

-1. Nunca

Fuente: Elaboración propia con base en los resultados estadísticos

La imagen 11, muestra que el 53\% de los empresarios Pymes de la muestra analizada opinan que siempre es necesaria la construcción de un sistema de seguimiento que ayude a lograr de forma efectiva la competitividad empresarial, el $47 \%$ opina que casi siempre es necesario. Los resultados muestran la importancia de la existencia de un sistema de seguimiento para lograr ventaja competitiva. 
Núm. 26

ISSN: 2007-8870

\section{http://revistainvestigacionacademicasinfrontera.com}

Imagen 12; Uso de estrategias para la competitividad empresarial

Uso de estrategias para enfrentar la competitividad de la empresa.

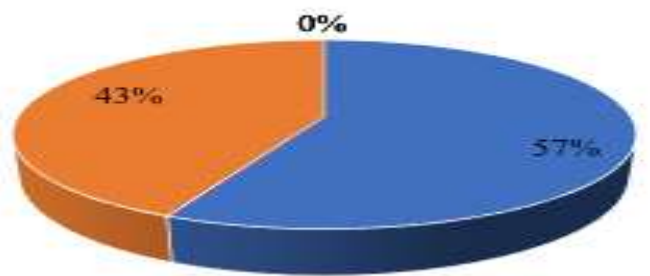

=5. Siempre

4. Casi siempre

- 3. Algunas veces

2. Casi nunca

-1. Nunca

Fuente: Elaboración propia con base en los resultados estadísticos

En los resultados de la imagen 12, se muestran las opiniones de los empresarios donde el $57 \%$ menciona que siempre es importante el uso de estrategias para enfrentar la competitividad de la empresa y el $43 \%$ opina que casi siempre es importante. Los resultados muestran que para estos empresarios la generación de estrategias empresariales solidas es la clave para la competitividad empresarial.

Imagen 13; Generación de innovación produce competitividad empresarial

La generación de Innovación que transfiere la universidad a las empresas, produce competitividad empresarial.

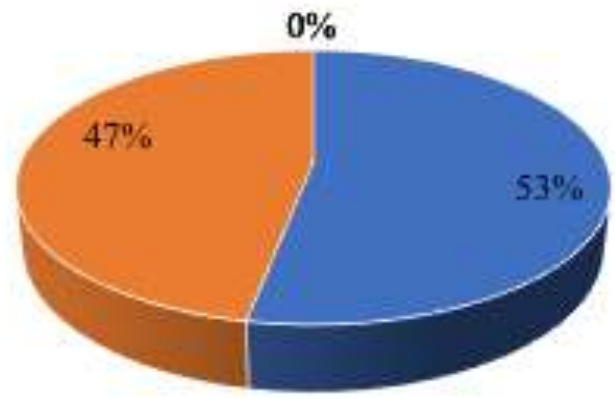

=5. Siempre

$=4$. Casi siempre

i. 3 . Algunas veces

2. Casi nunca

1. Nunca

Fuente: Elaboración propia con base en los resultados estadísticos 


\section{http://revistainvestigacionacademicasinfrontera.com}

Los resultados de la imagen 13 muestra que el 52\% de los empresarios opinan que la generarción de innovación que transfiere la universidad a las empresas, produce siempre competitividad empresarial y el $47 \%$ opina que casi siempre. Lo anterior hace ver que el empresario está consciente de que importancia que reviste para sus negocios la generación y aplicación de innovación en sus negocios.

\section{Conclusiones e implicaciones sociales y empresariales}

El formar alianzas con socios que cuenten con recursos económicos estratégicos para el desarrollo de tecnología y la puesta al mercado; así como el proceso de fabricación, comercialización y distribución de la innovación, son algunas de las razones para la transferencia de tecnología. La transferencia de tecnología tiene importancia para quien la genera como para quien la aplica ya que favorece la competitividad de las empresas, entendiendo que con la adquisición de nuevas tecnologías se podrán desarrollar mejores productos y servicios a costos reducidos. Igualmente, puede ser una gran aliada para la obtención de recursos en las distintas etapas del desarrollo de la innovación; o bien permitiendo la recuperación de la inversión efectuada, que por lo general conllevan grandes sumas de dinero.

Los primeros resultados obtenidos en la investigación muestran que en la variable 1; generación del conocimiento, más del $90 \%$ de los empresarios están conscientes de la importancia que tiene la vinculación Universidad-Pyme como fuente de innovación para sus negocios, saben que los conocimientos que tienen las personas al interior de la universidad son imprescindibles y útiles para generar proyectos productivos que ayuden a sus negocios a lograr competitividad empresarial.

En referencia a la variable 2, aplicación del conocimiento, los primeros resultados y de acuerdo a las opiniones de más del 60\% de los empresarios Pymes de la muestra analizada, están conscientes de la importancia de la innovación hoy en día y preocupados por lograr ventaja 


\section{http://revistainvestigacionacademicasinfrontera.com}

competitiva, lo que les hace estar conscientes de que deben siempre de definir una estrategia o plan de I+D en sus negocios. Para estos empresarios es importante contar con oportunidades de participación en proyectos de I+D ya que es una fuente de innovación y conocimiento para la empresa y les permita dotar a sus negocios de la fuerza necesaria para enfrentar los actuales mercados globalizados. Basado en lo anterior, el empresario opina que la transferencia de tecnología es gran ayuda para establecer estrategias para mejorar sus sistemas de gestión internos que le permitan enfrentar la competencia de los mercados y obtener ventaja competitiva.

En la variable 3, competitividad empresarial, los primeros resultados de la investigación hacen ver que el 53\% de los empresarios Pymes de la muestra analizada opinan que siempre es necesaria la construcción de un sistema de seguimiento que ayude a lograr de forma efectiva la competitividad empresarial y, para lograrlo es necesario crear estrategias empresariales sólidas basadas en la generación y aplicación de innovación en sus negocios.

Una de los objetivos del Plan de Desarrollo Institucional de la Universidad de Sonora está centrado en el desarrollo de proyectos de investigación científica orientados a apoyar el desarrollo económico, social y cultural del estado y del país y sus objetivos específicos es direccionar la elaboración de proyectos de investigación hacia la innovación y el desarrollo científico, tecnológico, humanístico y social, que tenga como prioridad la detección expedita de las necesidades sociales y económicas del estado y que contribuyan al avance de la competitividad y desarrollo sostenible de los distintos sectores de la sociedad.

Con base en lo anterior, se cumple con el objetivo planteado en la presente investigación porque se pone de manifiesto la importancia que tiene para los empresarios de las Pymes de Hermosillo, Sonora reconocer la influencia de la transferencia de tecnología Universidad-Pymes en la competitividad empresarial porque es indudable que el desarrollo científico y tecnológico es un factor potencial para el crecimiento económico y social de cualquier país. De manera que, el valor productivo que está inmerso en la transferencia de tecnología se basa en el desarrollo de nuevos conocimientos e innovaciones, producto de procesos de investigación orientados a incrementar la capacidad tecnológica que ayudará a la Pyme a lograr competitividad empresarial. 


\section{http://revistainvestigacionacademicasinfrontera.com}

De la misma manera es necesario dejar claro que la metodología aplicada en este estudio podrá ser utilizada y ampliada en el proceso de la misma investigación y en otros casos. Los primeros resultados de este trabajo servirán para orientar a las Pymes hacia la reflexión sobre la importancia de la aplicación del conocimiento para la elaboración de planes efectivos de capacitación orientada a este concepto que ayude a lograr competitividad empresarial de sus negocios. También serán útiles para un estudio cuantitativo posterior más amplio, el cual abarcará más empresas locales y permitirá obtener información sobre otras poblaciones en el estado o nacionales.

\section{Referencias bibliográficas}

- Aguilar Martínez, M. y Martínez Alvarado, K. (2013) :"Las PYMES ante el proceso de la globalización", en Observatorio de la Economía Latinoamericana, $\mathrm{N}^{\circ} 185$. Texto completo en http://www.eumed.net/cursecon/ecolat/mx/2013/pymes.html.

- Algieri, B., Aquino, A., \& Succurro, M. (2011). Technology transfer offices and academic spin-off creation: the case of Italy. Recuperado a partir de http://www.scopus.com/inward/record.url?eid=2-s2.081555231679\&partnerID=40\&md5=9271600f45dc42a773ec23d5b095be45

- Assink, M. (2006). Inhibitors of Disruptive Innovation Capability. European Journal of Innovation Management.

- Camacho Caicedo, A. (2008). Impacto de la gestion tecnologica en la competitividad de las pymes caleñas. Entramado, Vol.4 No. 1, pp. 8-16.

- Canales, D., Glover, A., González, M., \& al, e. (2008). Apoyando la Innovación en las PYMES: El estado del arte. FUNDACIÓN IDEA/USAID, 1-115. 


\section{http://revistainvestigacionacademicasinfrontera.com}

- CONACYT. (2011). Consejo Nacional de Ciencia y Tecnología. Obtenido de www.conacyt.gob.mx, consultado el 20 de abril de 2017.

- Cortes, Peñaloza (2011). Perspectiva tecnológica, Transferencia Tecnológica en Pymes.http://www. fpnt.org.mx/boletín/Abril 2011.

- Gómez M. (2007). El futuro de las PyME’s en el marco del T.L.C. en Las MIPyME’s en Latinoamérica, Estudios e Investigaciones en la Organización Latinoamericana de Administración. México: Universidad de Guanajuato

- Hernández, J. (2007). "Implementing strategically aligned performance measurement in small firms”, International Journal Production Economics, 106: pp. 393-408.

- Loaiza, Giugni, Monguet, (2009) Versión impresa ISSN 1316-4821 uct v.13 n.51 Puerto Ordaz jun.

- Markatou, Maria. (2012), The Role and the Importance of the Greek SMEs in the Production of Innovation', National and Kapodistrian University of Athens, Technological Education Institute of Larissa, Larissa, Greece.

- Pedraza E. y Velázquez J. (2013). Oficinas de Transferencia Tecnológica en las Universidades como Estrategia para Fomentar la Innovación y la Competitividad. Caso: Estado de Hidalgo, México. Journal of technology Management \& Innovation 2013, vol.8, núm. 2, pp.221-234. Universidad Alberto Hurtado, Santiago de Chile.

- Ruiz Durán,C. (2008). México: Geografía económica de la innovación. Comercio Exterior, Vol. 58, Núm. 11.

- Ruiz González, M. 1 y Mandado Pérez, E. (1989). La innovación tecnológica y su gestión. Editorial Productica, Marcombo S.A., España.

- Sepúlveda, M. (2005). Políticas e instrumentos para mejorar la gestión ambiental en la PyME y promover su oferta de bienes y servicios ambientales. [Documento en línea]. Proyecto CEPAL/GTZ GER/01/038, Chile. Disponible en URL: http://www.cepal.org/ publicaciones/xml/4/23244/112.pdf [Consulta 25 de Marzo de 2016]. 


\section{http://revistainvestigacionacademicasinfrontera.com}

- Solleiro y Castañón R. (2008), Gestión Tecnológica: Conceptos y prácticas. Editorial México, primera edición 2008, México D.F.

- Solleiro, J. (2008). Gestión Tecnológica: conceptos y prácticas, UNAM, México.

- Soto, Eduardo (2003). Las PYMES ante el desafío del siglo XXL: los nuevos mercados globales, editorial: Thompson pág. 2.

- Storey, J. (2000): The management of innovation problem. International Journal of Innovation Management, vol. 4, no. 3, pp. 347-369.

- Vargas, J. y Guillen, I. (2005). Los procesos de transformación estratégica en relación con la evolución de las organizaciones. Estudios Gerenciales, Vol. 94, pp. 65-80. 